Parameter Scaling to Produce Different Charged-Particle Beam-Transport Systems Having ldentical Equations of Motion

E. A. Wadlinger

\title{
MASTER
}

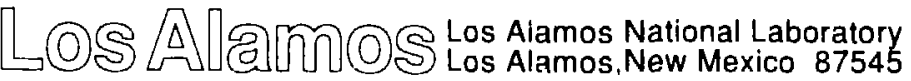




\section{Parameter scaling to Produce Different Charged-Particle Beam-Transport Systems HaVing IDENTICal EQuations Of Motion}

\section{by}

E. A. Wadlinger

\section{SUMMARY}

Designs are frequently required for charged-particle optics channels to transport space-charge (Coulomb) forcedominated beams for use with accelerators under design or construction. It is often desirable to experimentally test a design using an existing accelerator having different parameters from the one under consideration. This paper shows how to scale a charged-particle transport design to make a model experiment for testing the design with an existing accelerator. Sometimes, with the proper choice of accelerator, the model experiment can be much less expensive than the target device.

By scaling variables (beam current and emittance, magnetic fields, etc.) in a way that produces certain invariant quantities, we obtain identical equations of motion for different charged-particle beam channels transporting different beams. The scaling relations take a transport channel design for one application having a given time structure (for instance, the time structure determined by the radio frequency of a beam bunching cavity), beam energy, current, etc., and determine an equivalent transport channel design for another device with different parameters. Therefore, we can predict particle and beam behavior in one situation by knowing it in another. Even growth in emittance will be modeled correctly in spite of the fact that the devices 
transport beams with different time structure, energy, and currents. The scaling relations, which are limited to nonaccelerated systems, include the following:

- relativistic motion,

- time-dependent (or independent) electric and magnetic external fields,

- self-forces given by the Poisson equation using a singleparticle charge distribution $\rho(\vec{r})$, and

- image-charge forces (properly adjusted by the scaled boundary conditions).

We work with the single-particle second-order differential equation of motion. In order to exactly model one system with another, the beam position, velocity distributions, and the external electromagnetic fleld distributions of the systems must have the same 'shape' when expressed in the scaled coordinates. The scaled systems will have both identical equations of motion and identical initial and boundary conditions.

The next section contains the derivation of the scaling relations. We then summarize the scaling relations, discuss their implications, and give one example to show their utility. Appendixes $A$ and $B$ are included to indicate how to modify PARMILA and other transport codes input to obtain scaled systems and to show how solenoids scale.

\section{SCALING-RELATION DERIVATION}

\section{Introduction}

We derive the scaling laws that determine the parameter space of charged-particle beam channels and beams that have nearly identical (within the approximations made beiow) single-particle equations of motion. Given the appropriate ruler, stop watch, and scaled boundary conditions, all scaled systems are indistinguishable from each other and have the sane relative time evolution.

The method of approach is to normalize the position and time using the scaling parameters (length $L$, time $\tau$ ) so that the normalized variables are dimensionless. The shapes of the various external electromagnetic fields, beam-charge density distributions, and velocity distributions are given in terms of the normalized, dimensionless variables. Because the dis'ributions are parametrized in this way, they are the same in all scaled systems. 
The charged-particle beams have an average velocity $v_{0}$ with individual particles having a velocity spread $\Delta \vec{v}$ about the average velocity. The ratio $\Delta \vec{v} / v_{0}$ distributions must be the same in all scaled systems (with different $v_{0}$ ) for the systems to be indistinguishable. It is common practice to characterize the beam-particle distribution in position and velocity space in terms of emittance. We define emittancs as the area occupied by particles in phase space $\left(u, u^{\prime}=\Delta v_{u} / v_{0} ; u=x, y, z\right)$. We will show how emittance scales between different transport systems.

In this paper, variables containing the subscript ' $n$ ' are normalized, dimensionless variables (length and time). Parameters containing the subscript ' $g$ ' are geometrical (form factor) quantities (charge-density distribution, etc.) parametrized by the normalized, dimensionless variables and are identical in all scaled systems.

In the next subsections, we write down the equation of motion and describe its parameters. We then list the two approximations used to derive the scaling laws, define the normalizations to permit the factorization of certain parameters into dimensioned variables and dimensionless geometric factors, and combine the equations in a form that permits easy identification of the scaling laws.

\section{Equation of Motion}

In this section, we write the equation of motion describing individual particle behavior and define all the parameters used in this equation. Each individual particle has its own equation of motion describing its time evolution where the individual equations differ because of the individual paricle interactions with other beam particles. However, all the equations scale identically and we, therefore, consider a single equation of motion. (We use SI units to formulate the equations of motion. However, the scaling rules are independent of the system of units.)

The single-particle equation of motion in the presence of external electromagnetic fields and space-charge fields resulting from the particle interactions is

$$
d \vec{P} / d t=e \vec{E}+e \vec{v} \times \vec{B},
$$

where e and $\vec{v}$ are the particle's charge and velocity, $\vec{E}$ and $\vec{B}$ are the electric and magnetic fields, and $\vec{P}$ is the particle momentum

$$
\vec{P}=m_{0} c \gamma \vec{\beta},
$$

where $m_{0}, \vec{\beta}$, and $\gamma$ are the particle's rest mass, velocity divided by the speed of light (c), and the total energy divided by the rest mass energy $\left[=\left(1-|\vec{\beta}|^{2}\right)^{-1 / 2}\right]$. 
We separate the magnetic and electric fields into external fields and seif-fields resulting from particle interactions and write

$$
\begin{aligned}
& \vec{E}=\vec{E}_{e x t}+\vec{E}_{C}, \\
& \vec{B}=\vec{B}_{e x t}+\vec{B}_{C},
\end{aligned}
$$

where

$$
\begin{array}{ll}
\vec{E}_{\text {ext }}=\vec{E}_{\text {ext }}(\vec{r}, t), & \text { external electric field; } \\
\vec{B}_{\text {ext }}=\vec{B}_{\text {ext }}(\vec{r}, t), & \text { external magnetic field; } \\
\vec{E}_{C}\left(\vec{r}_{2}, t\right)=\frac{1}{4 \pi \epsilon_{0}} \int \frac{\rho_{1} \vec{r}_{21} d V_{1}}{\left|\vec{r}_{21}\right|^{3}}, & \text { charge-induced electric field; } \\
\vec{B}_{C}\left(\vec{r}_{2}, t\right)=\frac{\mu_{0}}{4 \pi} \int \frac{\vec{J}_{1} \times \vec{r}_{21} d V_{1}}{\left|\vec{r}_{21}\right|^{3}}, & \text { current-induced maginetic field; } \\
\rho_{1}=\rho\left(\vec{r}_{1}, t\right), & \text { charge-density distribution; }
\end{array}
$$

and

$$
\vec{J}_{1}=\vec{J}\left(\vec{r}_{1}, t\right), \quad \text { current-density distribution }
$$

are all functions of position and time. [The parameter $\vec{r}_{21}=\left(\vec{r}_{2}-\vec{r}_{1}\right)$ in the above equations.]

The center of mass of a beam distribution moves with the velocity $\vec{v}_{0}=v_{0} \hat{k}$, where $\hat{k}$ is the unit vector in the axial direction. (Also indicated by the subscript $\|$. Directions perpendicular to the axial direction are denoted by the subscript 1 .) There is a relative velocity spread of the particles in the beam distribution that is parametrized by the emittance (giving the particle distribution in position and velocity phase space) and emittance orientation in phase space. This velocity spread is affected by the space-charge forces. The individual particle velocity, used in the equation of motion Eq. (1), is

$$
\vec{v}=v_{0}\left(\hat{k}+\left.\frac{\Delta \vec{v}}{v_{0}}\right|_{e m i t}+\left.\frac{\Delta \vec{v}}{v_{0}}\right|_{s c}\right),
$$

where the subscript 'emit' denotes the particle velocity resulting from the beam emittance and the subscript ' $s c$ ' denotes the space-charge-induced particle velocity resulting from changes in the Coulomb energy associated with the beam distribution (see below). 
We have defined the single particle equation of motion and its parameters. In the next section, we discuss the approximations used to calculate the above quantities.

\section{Approximations}

We make two approximations. The first is the paraxial approximation

$$
\vec{v} \approx|\vec{v}| \hat{k}=\beta c \hat{k}
$$

which is used for calculating the time derivatives on the left-hand side of the equation of motion Eq. (1) and for calculating the forces resulting from the beam-current-induced magnetic fields. This is a good approximation in most beam-transport systems (see EXAMPLE section). [Note that $\vec{v}$ in Eq. (12) is the particle velocity.]

The second approximation relates the beam current density to the charge density by assuming that

$$
\vec{J}=v_{0} \rho k \text {. }
$$

This approximation is an extension of the paraxial approximation and assumes that the velocity spread between particles is not important when caiculating beam-particle-induced magnetic forces. This is an extremely accurate approximation for most beam-transport applications and is used to evaluate Eq. (8).

\section{Normalizations}

Physics does not depend on the choice of units. We write the physical quantities that we wish to scale (time and position) in dimensionless units by introducing the length and time-scaling parameters $L$ and $\tau$ (in SI units, the dimension of $L$ is in meters and the dimension of $\tau$ is in seconds). We denote the normalized, dimensionless variables with the subscript $n$ and write

$$
\vec{r}=L \vec{r}_{n} \quad \text { and } \quad \mathrm{t}=\tau \mathrm{t}_{\mathrm{n}}
$$

then

$$
\frac{d}{d \vec{r}}=\frac{1}{L} \frac{d}{d \vec{r}_{n}} \quad \text { and } \quad \frac{\mathrm{d}}{\mathrm{dt}}=\frac{1}{\tau} \frac{\mathrm{d}}{\mathrm{dt}_{\mathbf{n}}}
$$

The relations

$$
d V=L^{3} d V_{n} \quad \text { and } \quad \mathrm{dA}=\mathrm{L}^{2} \mathrm{dA}_{\mathrm{n}}
$$


for the differential volume and area follow from Eq. (14). Because the parameters we scale are independent of the coordinate system, we work with Cartesian coordinates.

Normalization of External Fields. We consider the form of the external electric and magnetic fields using normalized, dimensionless parameters. The nethod of approach for evaluating the electric and magnetic fields is the same; therefore, we evaluate the electric field and state the results for the magnetic field.

We factor the external fields into two parts. One factor (denoted by the subscript ' 0 ') determines the dimensioned field strength at some fixed equivalent point (perhaps on a pole tip) in all scaled systems. The other factor is dimensionless and identical in all scaled systems and represents the form or shape of the external field and is parametrized in terms of the normalized, dimensionless variables $\left(\vec{r}_{n}, t_{n}\right)$.

We write the electric field in Eq. (5) as

$$
\vec{E}_{e x t}(\vec{r}, t)=E_{0} \vec{E}_{g}\left(\frac{\vec{r}}{L}, \frac{t}{\tau}\right)=E_{0} \vec{E}_{g}\left(\vec{r}_{n}, t_{n}\right)
$$

where $\vec{E}_{g}$ is a dimensionless geometric (shape or form) factor (hence the subscript $g$ ), which is the same in all physically equivalent systems. (The factor $E_{g}$ can be represented by a Taylor series expansion in $\vec{r}_{n}$ and $t_{n}$.) We normalize $\vec{E}_{g}$ so that

$$
\vec{E}_{g}\left(\vec{r}_{n_{0}}, t_{n_{0}}\right)=1
$$

in all scaled systems at a fixed $\left(\vec{r}_{n_{0}}, t_{n_{0}}\right)$. (The exact values chosen for $\vec{r}_{n_{0}}$ and $t_{n_{0}}$ are not important.) The quantity $E_{0}$ determines the electric field strength at $\left(\vec{r}_{n_{0}}, t_{n_{0}}\right)$ and will vary between systems as determined by the scaled equations of motion.

Similarly, we let

$$
\vec{B}_{e x t}(\vec{r}, t)=B_{0} \vec{B}_{g}\left(\vec{r}_{n}, t_{n}\right)
$$

where

$$
\vec{B}_{g}\left(\vec{r}_{n_{n}}, t_{n_{0}}\right)=1
$$

Normalization of Charge-Density Integrals. Three different integrals involving the charge density appear in the equations of motion. These integrals are

$$
\vec{S}_{1}=\int_{V} \frac{\rho \vec{r} d V}{|\vec{r}|^{3}}
$$




$$
S_{2}=\int_{V} \frac{\rho d V}{|\vec{r}|}
$$

and

$$
S_{3}=\int_{A} \rho d A
$$

where Eqs. (21) and (22) are volume integrals and Eq. (23) is an area integral proportional to the particle flux. We rewrite these integrals so that they are proportional to dimensionless, geometrically invariant (between systems) integrals.

We treat the charge density $\rho$ as we did the external fields and write

$$
\rho(\vec{r}, t)=\rho_{0} \rho_{g}\left(\vec{r}_{n}, t_{n}\right),
$$

where $\rho_{g}$ is a shape or form factor describing the charge distribution and is normalized such that

$$
\rho_{g}\left(\vec{r}_{n_{0}}, t_{n_{0}}\right)=1
$$

with $\vec{r}_{n_{0}}$ and $t_{n_{0}}$ the same in all scaled systems (the exact values are again not important). The quantity $\rho_{0}$ determines the magnitude of the charge and will vary between systems as determined by the scaled equation of motion. We will later relate $\rho_{0}$ to the total beam current $I_{0}$.

We calculate $\vec{S}_{1}$ by substituting the normalized volume integral [Eq. (16)] and the charge density [Eq. (24)] into Eq. (21) to obtain

$$
\begin{aligned}
\vec{S}_{1} & =\int_{V_{n}} \frac{\rho_{0} \rho_{g}\left(L \vec{r}_{n}\right)\left(L^{3} d V_{n}\right)}{\left|L \vec{r}_{n}\right|^{3}} \\
& =\rho_{0} L \int_{V_{n}} \frac{\rho_{g} \vec{r}_{n} d V_{n}}{\left|\vec{r}_{n}\right|^{3}} .
\end{aligned}
$$

We define

$$
\vec{S}_{1 g}\left(\vec{r}_{n_{2}}, t_{n}\right)=\int \frac{\rho_{g} \vec{r}_{n_{21}} d V_{n_{1}}}{\left|\vec{r}_{n_{21}}\right|^{3}}
$$

which is required to be the same in all scaled systems. Note that the integral in Eq. (27) contains only dimensionless, normalized, variables and geometric factors. Combining Eqs. (26) and (27) gives 


$$
\vec{S}_{1}=\int_{V} \frac{\rho \vec{r} d V}{|\vec{r}|}=\rho_{0} L \vec{S}_{1 g}
$$

By defining

$$
S_{2 g}=\int_{V_{n}} \frac{\rho_{g} d V_{n}}{\left|\vec{r}_{n}\right|}
$$

and

$$
S_{3 g}=\int_{A_{n}} \rho_{g} d A_{n}
$$

we can show, using the above procedure, that

$$
S_{2}=\int_{V} \frac{\rho d V}{|\vec{r}|}=\rho_{0} L^{2} S_{2 g}
$$

and

$$
S_{3}=\int_{A} \rho d A=\rho_{0} L^{2} S_{3 g} .
$$

It is important to note that the integrals $\vec{S}_{1 g}, S_{2 g}$, and $S_{3 g}$ are required to be the same in all scaled systems. Equivalently, the distribution of charge expressed in normalized coordinates must be the same for all scaled systems.

\section{Time Derivatives}

We evaluate the time derivative of the momentum in the left-hand side of the equation of motion [Eq. (1)] using the momentum defined in Eq. (2) and the paraxial approximation [Eq. (12)]. We calculate

$$
\begin{aligned}
d \vec{P} / d t & =m_{0} c\left(\dot{\vec{\beta}}_{\perp} \gamma+\dot{\vec{\beta}}_{\|} \gamma^{3}\right) \\
& =m_{0}\left(\gamma \frac{d^{2} \vec{r}_{\perp}}{d t^{2}}+\gamma^{3} \frac{d^{2} \vec{r}_{\|}}{d t^{2}}\right),
\end{aligned}
$$

where the 'dots' denote time derivatives. Use the normalizations of Eq. (14) to obtain

$$
d \vec{P} / d t=\frac{m_{0} L}{\tau^{2}}\left(\gamma \frac{d^{2} \vec{r}_{n_{\perp}}}{d t_{n}^{2}}+\gamma^{3} \frac{d^{2} \vec{r}_{n_{\|}}}{d t_{n}^{2}}\right)
$$


where ' $\mathrm{L}$ ' is time independent (nonaccelerated systems). The derivatives on the right-hand side of Eq. (34) are expressed in normalized, dimensionless quantities and are required to be the same in all scaled systems. This requirement determines the scaling laws.

\section{Velocity Spread $\Delta \vec{v} / v_{0}$ and Emittance}

There is a velocity spread, in Eq. (11), resulting from the beam emittance that can change in time because of the space-cisarge forces. This velocity spread, which we discuss here, produces chromatic aberrations in the $\vec{v} \times \vec{B}$ term in the equation of motion Eq. (1).

Space-charge forces produce a change in velocity spread that is proportional, through energy conservation, to the space-charge potential function,

$$
\begin{aligned}
\phi & =\frac{1}{4 \pi \epsilon_{0}} \int \frac{\rho_{1} d V_{1}}{\left|\vec{r}_{21}\right|} \\
& =\rho_{0} L^{2} \frac{S_{2 g}}{4 \pi \epsilon_{0}},
\end{aligned}
$$

where we have used the normalized integral of Eq. (31). (Remember that $S_{2 g}$ is a dimensionless form factor that is identical in all scaled systems.) We use the free-space Green's function to calculate the space-charge potential function and ignore the image charges that represent conducting wall boundary conditions. However, when perfectly conducting walls are included, the appropriate scaled image charges are automatically obtained because of the scaling of the wall boundary positions.

The energy spread $W$ is related to the space-charge potential function through $\mathrm{W}+\mathrm{q} \phi=$ const. The change in energy spread $\Delta E=\Delta W$ is proportional to the change in $\phi$, which is proportional to the change in $\mathrm{S}_{2 g}$. Therefore,

$$
\Delta E=-\rho_{0} L^{2} \frac{q \Delta S_{2 g}}{4 \pi \epsilon_{n}}
$$

We obtain from Eq. (2), using the paraxial approximation and considering only longitudinal velocity spreads,

$$
\left.\frac{\Delta \vec{v}_{\|}}{v_{0}}\right|_{s c}=\left.\frac{\Delta \vec{P}_{\|}}{\gamma^{2}|\vec{P}|}\right|_{s c} .
$$

From

$$
E^{2}=P^{2} c^{2}+\left(m_{0} c^{2}\right)^{2}
$$


we obtain

$$
\left.\frac{\Delta \vec{P}_{\|}}{|\vec{P}|}\right|_{s c}=\frac{\Delta E \hat{k}}{\gamma \beta^{2} m_{0} c^{2}} .
$$

Combining Eqs. (35) through (39) gives

$$
\left.\frac{\Delta \vec{v}_{\|}}{v_{0}}\right|_{s c}=-\frac{\rho_{0} L^{2}}{m_{0} \beta^{2} \gamma^{3}} \frac{q \Delta S_{2 g} \hat{k}}{4 \pi \epsilon_{0} c^{2}} .
$$

Note that the term $\left[q \Delta S_{2 g} /\left(4 \pi \epsilon_{0} c^{2}\right)\right]$ is the same in all scaled systems.

We require that $\left.\frac{\Delta \bar{v}}{v}\right|_{e m i t}$ be the same in all scaled systems so that these systems are indistinguishable. This condition determines the emittance scaling. The equations of motion then guarantee that the systems remain indistinguishable in time.

The unnormalized emittance is defined as

$$
\epsilon_{l a b}=\left(1+\alpha^{2}\right)^{-1 / 2} U^{\prime} U,(U=X, Y, Z),
$$

where $U$ is the beam size (maximum or rms, the definition is not important so long as it is the same in all scaled systems; also, the beam orientation in phase space, represenied by the dimensionless parameter $\alpha$, is not important so long as it is the same in all scaled systems), and $U^{\prime}$ is the beam divergence $\left(\Delta v_{U} / v_{0}\right)$. This emittance written in terms of normalized, dimensiorless parameters is

$$
\epsilon_{l a b}=\left(1+\alpha^{2}\right)^{-1 / 2} L U^{\prime} U_{n},
$$

where $\left(U=L U_{n}\right)$. Accelerator designers often work with a normalized emittance $\epsilon_{\text {norm }}$, which may be preserved during acceleration (note that this emittance is not dimensionless and will differ between scaled systems). This emittance is defined as

$$
\epsilon_{\text {norm }}=\beta \gamma \frac{\Delta P}{P} U\left(1+\alpha^{2}\right)^{-1 / 2}=\beta \gamma^{p} L U^{\prime} U_{n}\left(1+\alpha^{2}\right)^{-1 / 2},
$$

where the power $p=1(3)$ for transverse (longitudinal) motion.

\section{Combined Space-Charge Electric and Magnetic Fields.}

In this section, we substitute the current density of Eq. (13) into Eq. (8) for the currentinduced magnetic field. We ignore the velocity spreads caused by emittance and spacecharge terms in Eq. (11) and calculate $\vec{v}_{0} \times \vec{B}_{C}$. We show, by combining $\vec{E}_{C}+\left(\vec{v}_{0} \times \vec{B}_{C}\right)$, 
that the effective longitudinal Coulomb force is not affected by the self-magnetic field while, as expected, the transverse Coulomb force is reduced by $1 / \gamma^{2}$.

Substitute Eq. (13) into Eq. (8) and use the paraxial approximation, ignoring all velocity spreads, to obtain

$$
\begin{aligned}
\vec{v} \times \vec{B}_{C} & \approx v_{0} \hat{k} \times \vec{B}_{C} \\
& \approx \frac{\mu_{0} v_{0}^{2} \hat{k} \times}{4 \pi} \int \frac{\hat{k} \times \rho_{1} \vec{r}_{21} d V_{1}}{\left|\vec{r}_{21}\right|^{3}} \\
& =-\beta^{2} \vec{E}_{C_{\perp}},
\end{aligned}
$$

where we have used $\mu_{0} \epsilon_{0}=1 / c^{2}$ and Eq. (7), which defines $\vec{E}_{C}$. Using Eq. (44) we obtain

$$
\vec{E}_{C}+\vec{v} \times \vec{B}_{C} \approx \vec{E}_{C_{\sharp}}+\frac{\vec{E}_{C_{\perp}}}{\gamma^{2}}
$$

Substitute the normalized integral of Eq. (28) in Eq. (7) to obtain

$$
\vec{E}_{C}=\rho_{0} L \frac{\vec{S}_{1 g}}{4 \pi \epsilon_{0}}
$$

\section{Current vs Charge Density}

We relate the charge density $\rho$ to the total beam current $I_{0}$. The paraxial approximation Eq. (13) and the normelized integral Eq. (32) are used to obtain

$$
I_{0}=\int v_{0} \rho d A=v_{0} \rho_{0} L^{2} S_{3 g}
$$

\section{The Normalized Equation of Motion}

The equations from the previous sections are combined to obtain the scaling rules. We start with the single-particle equation of motion, Eq. (1), for a charged particle that interacts with external electromagnetic fields as well as particles in the beam. Substitute into this equation: Eq. (34) containing the normalized time derivative of the momentum; Eqs. (17) and (19) containing the external electric and magnetic fields; Eqs. (45) and (46), which describe the electric and magnetic forces resulting from other charged particles in the beam; Eqs. (11) and (40), which express the velocity terms; and Eq. (47), which relates the total beam current to the charge density. The equation of motion becomes 


$$
\begin{aligned}
\frac{m_{0} L}{\tau^{2}}\left(\gamma \frac{d^{2} \vec{r}_{n_{\perp}}}{d t_{n}^{2}}\right. & \left.+\gamma^{3} \frac{d^{2} \vec{r}_{n_{\|}}}{d t_{n}^{2}}\right)=e\left[E_{0} \vec{E}_{g}\right. \\
& +v_{0} B_{0}\left(1+\left.\frac{\Delta v_{\|}}{v_{0}}\right|_{e m i t}-\frac{I_{0}}{\pi_{0 \cdot 0} \beta^{3} \gamma^{3}} \frac{q \Delta S_{2 g}}{4 \pi \epsilon_{0} c^{3} S_{3 g}}\right) \hat{k} \times \vec{B}_{g} \\
& \left.+\frac{I_{0}}{\beta L} \frac{\vec{S}_{1 g_{\|}}}{4 \pi \epsilon_{0} c S_{3 g}}+\frac{I_{0}}{\beta L \gamma^{2}} \frac{\vec{S}_{1 g_{\perp}}}{4 \pi \epsilon_{0} c S_{3 g}}\right] .
\end{aligned}
$$

The conventions used in this equation are that the subscripts ' $n$ ' denote dimensionless, normalized variables; subscripts ' $g$ ' denote dimensionless, geometric (shape or form) factors that are the same in all scaled systems; and $\hat{k}$ is a vector in the axial direction. We separate Eq. (48) into transverse and longitudinal components and rearrange terms to get

$$
\begin{aligned}
\frac{d^{2} \vec{r}_{n_{\perp}}}{d t_{n}^{2}}= & \frac{e \tau^{2}}{m_{0} L \gamma}\left[E_{0} \vec{E}_{g \perp}+v_{0} B_{0}\left(1+\left.\frac{\Delta v_{\|}}{v_{0}}\right|_{e m i t}-\frac{I_{0}}{m_{0} \beta^{3} \gamma^{3}} \frac{q \Delta S_{2 g}}{4 \pi \epsilon_{0} c^{3} S_{3 g}}\right) \hat{k} \times \vec{B}_{g}\right. \\
& \left.+\frac{I_{0}}{\beta L \gamma^{2}} \frac{\vec{S}_{1 g_{\perp}}}{4 \pi \epsilon_{0} c S_{3 g}}\right] \\
= & \frac{\tau^{2} E_{0}}{m_{0} L \gamma} \vec{A}_{1 \perp}+\frac{\tau^{2} \beta B_{0}}{m_{0} L \gamma}\left(1+\left.\frac{\Delta v_{\|}}{v_{0}}\right|_{e m i t}+\frac{I_{0}}{m_{0} \beta^{3} \gamma^{3}} A_{3}\right) \vec{A}_{2 \perp} \\
& +\frac{I_{0} \tau^{2}}{m_{0} L^{2} \beta \gamma^{3}} \vec{A}_{4 \perp}
\end{aligned}
$$

and

$$
\begin{aligned}
\frac{d^{2} \vec{r}_{n_{\|}}}{d t_{n}^{2}} & =\frac{e \tau^{2}}{m_{0} L \gamma^{3}}\left(E_{0} \vec{E}_{g \|}+\frac{I_{0}}{\beta L} \frac{\vec{S}_{1 g_{\|}}}{4 \pi \epsilon_{0} c S_{3 g}}\right) \\
& =\frac{\tau^{2} E_{0}}{m_{0} L \gamma^{3}} \vec{A}_{1 \|}+\frac{I_{0} \tau^{2}}{m_{0} L^{2} \beta \gamma^{3}} \vec{A}_{4 \|}
\end{aligned}
$$

where

$$
\begin{aligned}
& \vec{A}_{1}=e \vec{E}_{g}, \\
& \vec{A}_{2}=e c \hat{k} \times \vec{B}_{g}, \\
& \vec{A}_{3}=\frac{-q \Delta S_{2 g}}{4 \pi \epsilon_{0} c^{3} S_{3 g}},
\end{aligned}
$$

and 


$$
\vec{A}_{4}=\frac{e \vec{S}_{1 g}}{4 \pi \epsilon_{0} c S_{3 g}}
$$

The parameters $A_{1-4}, \Delta \vec{v} /\left.v_{0}\right|_{\text {emit }}$ (by definition) and the normalized derivatives on the left-hand sides of Eqs. (49) and (50) are the same in all scaled systems. Therefore, the functions $D_{1-5}$

$$
\begin{aligned}
& D_{1}=\frac{\tau^{2} E_{0}}{m_{0} L \gamma^{p}}, \\
& D_{2}=\frac{\tau^{2} \beta B_{0}}{m_{0} L \gamma}, \\
& D_{3}=\frac{I_{0}}{m_{0} \beta^{3} \gamma^{3}}, \\
& D_{4}=\frac{\tau^{2} I_{0}}{m_{0} L^{2} \beta \gamma^{3}}
\end{aligned}
$$

and

$$
D_{5}=\frac{D_{3}}{D_{4}}=\frac{L^{2}}{\beta^{2} \tau^{2}}
$$

are also the same in all scaled systems. Equation (59) gives the scaling relation between beam velocity, time, and length. Once this relation is established, Eq. (57) scales the current and mass, Eq. (56) scales the transverse magnetic pole-tip field, and Eq. (55) scales the electric field [the power $\mathrm{p}=1$ (3) for transverse (longitudinal) electric fields].

\section{SCAI.ING RELATIONS}

We summarize the results derived in the previous sections. The parameters in the equation of motion were factored into the following categories:

- normalized, dimensionless variables (normalized length and time),

- fixed, dimensionless geometrical coordinate-system-independent parameters unaffected by the scaling (space-charge moment distributions, relative magnetic multipole distributions, etc.), and

- dimensioned parameters that change because of the scaling (emittance, current, electric and magnetic pole-tip field magnitudes).

We found that the equation of motion is unchanged under a class of transformations of the third set of parameters [scaling relations Eqs. (55) through (59)].

The scaling relations, obtained by rearranging Eqs. (55) through (59), are (the subscript 'S1' denotes system 1 and 'S2', system 2) 


$$
\begin{array}{ll}
\frac{\vec{x}_{S 2}}{L_{S 2}}=\frac{\vec{x}_{S 1}}{L_{S 1}}, & \text { length scaling; } \\
\frac{t_{S 2}}{\tau_{S 2}}=\frac{t_{S 1}}{\tau_{S 1}}, & \text { time scaling; } \\
\frac{v_{S 2} \tau_{S 2}}{L_{S 2}}=\frac{v_{S 1} \tau_{S 1}}{L_{S 1}}, & \text { average velocity scaling; } \\
\frac{I_{S 2}}{m_{0_{S 2}} \gamma_{S 2}^{3} \beta_{S 2}^{3}}=\frac{I_{S 1}}{m_{0_{S 1}} \gamma_{S 1}^{3} \beta_{S 1}^{3}}, & \text { current and mass scaling; } \\
\frac{E_{0 S 2} L_{S 2}}{m_{0_{S 2}} \gamma_{S 2}^{p} \beta_{S 2}^{2}}=\frac{E_{0_{S 1}} L_{S 1}}{m_{0_{S 1}} \gamma_{S 1}^{p} \beta_{S 1}^{2}}, & \text { electric pole-tip scaling; } \\
\frac{B_{0_{S 2}} L_{S 2}}{m_{0_{S 2}} \gamma_{S 2} \beta_{S 2}}=\frac{B_{0 S 1} L_{S 1}}{m_{0_{S 1}} \gamma_{S 1} \beta_{S 1}}, & \text { transverse magnetic pole-tip scaling; }
\end{array}
$$

and

$$
\frac{\epsilon_{\text {norm }}}{\beta_{S 2} \gamma_{S 2}^{p} L_{S 2}}=\frac{\epsilon_{n o r m s 1}}{\beta_{S 1} \gamma_{S 1}^{p} L_{S 1}}, \quad \text { normalized emittance scaling }
$$

where $L$ is a scaling length, $\vec{x}$ is a distance, $I$ is the current, $v$ is the beam velocity, $\gamma$ is the total beam energy divided by the beam's rest mass energy, $t$ is the time, $\tau$ is the time normalization, $E_{0}$ is an electric pole-tip field, $B_{0}$ is a magnet pole-tip field, $\epsilon_{\text {norm }}$ obtained from Eq. (43) is the normalized emittance, $m_{0}$ is the particle rest mass, and $p$ is a power ( 1 or 3 ) for (transverse or longitudinal) motion. Equation (60) scales length, Eq. (61) scales time, Eq. (62) scales velocity, Eq. (63) scales beam current, Eq. (64) scales the electric pole-tip field, Eq. (65) scales the magnet pole-tip field, and Eq. (66) scales normalized emittance.

Exactly which variables in Eqs. (60) through (66) are independent and dependent variables will be dictated by circumstances. For example, Eq. (62) relates time, length, and velocity. If the energy and time structure are defined, Eq. (62) determines the length scale ratio.

The plasma period scales as Eq. (61). The plasma frequency scales as the square-root of the particle density. Substituting Eq. (47) into Eq. (58) shows that the particle density scales like $1 / \tau^{2}$.

We show in Appendix B that the solenoid field strength scales as Eq. (65). 


\section{EXample: Beam DyNamics Comparison Between A 5-MeV AND 100-MeV BEAM-EXPANSION TELESCOPE}

Assume that we wish to experimentally model a beam-expanding telescope designed to mate to the downstream end of a 100-MeV, 1700-MHz proton linear accelerator (linac L1). We study this case because the beam makes a transition from an emittance-dominated beam to a space-charge-dominated beam during the expansion process [1-2]. The performance of this device is also sensitive to errors in transport-element field strength and beam emittance and current.

This telescope will expand, by a factor of $10^{3}$, a $0.2-\mathrm{A}$ current beam having a normalized transverse rms emittance of $1.0 \times 10^{-7} \pi \cdot \mathrm{m} \cdot \mathrm{rad}$ and a normalized longitudinal rms emittance of $1.4 \times 10^{-7} \pi \cdot \mathrm{m} \cdot \mathrm{rad}$ [see Eq. (43)]. The telescope contains a quadrupole triplet eyepiece followed by an rf longitudinal-momentum compaction cavity, a beam-expansion drift space, and a four-element quadrupole objective lens.

We assume that a $5-\mathrm{MeV}, 425-\mathrm{MHz}$ proton linac (L2) test stand is available to do a modeling experiment of the above telescope design. We use the scaling laws to determine the telescope and beam parameters required to perform an exact modeling experiment using this test stand.

The time scaling is determined by the if time period of the two linacs and the velocity scaling, by the output energy. We use Eq. (62) to calculate the length scales and Eqs. (63) through (66) to give the current, electric pole tip, magnetic pole tip, and normalized emittance scaling. Table I compares the parameters of the two devices. Column 1 of the table lists the device; column 2 , the rf frequency $\left(f_{0}\right)$; column 3 , the beam velocity/velocity of light; column 4, the length scale size relative to $L 1$; column 5 , the beam current; column 6 , the normalized rms transverse emittance; column 7, the pole-tip magnetic field strength ratio to $\mathrm{L} 1$; and column 8 , the electric pole-tip field strength ratio to $\mathrm{L} 1$.

\begin{tabular}{|cccccccc|}
\hline \multicolumn{6}{|c|}{ Table I. } & Parameters for Scaled Experiments & \\
\hline & $\begin{array}{c}f_{0} \\
\left(\times 10^{6}\right.\end{array}$ & $\mathrm{L}$ & $\mathrm{I}$ & $\begin{array}{c}\varepsilon_{T n} \\
\left(\times 10^{-7}\right.\end{array}$ & $\mathrm{B}_{0 r}$ & $\begin{array}{c}E_{0 \|} \\
\text { Device }\end{array}$ \\
$\mathrm{Hz})$ & $\beta$ & (relative) & $(\mathrm{A})$ & $\mathrm{m} \cdot \mathrm{rad})$ & (relative) & (relative) \\
\hline $\mathrm{L} 1$ & 1700 & 0.43 & 1.0 & 0.20 & 1.00 & 1.0 & 1.0 \\
$\mathrm{~L} 2$ & 425 & 0.10 & 0.96 & 0.0021 & 0.21 & 0.23 & 0.045 \\
\hline
\end{tabular}

Figure 1 presents the results of PARMILA* simulations. Note that the phase-space plots for the two cases are nearly identical. There is a factor of 3.4 increase in emittance by the

* PARMILA, a widely used linear accelerator and beam transport simulation program developed at Los Alamos National Laboratory (undocumented). 

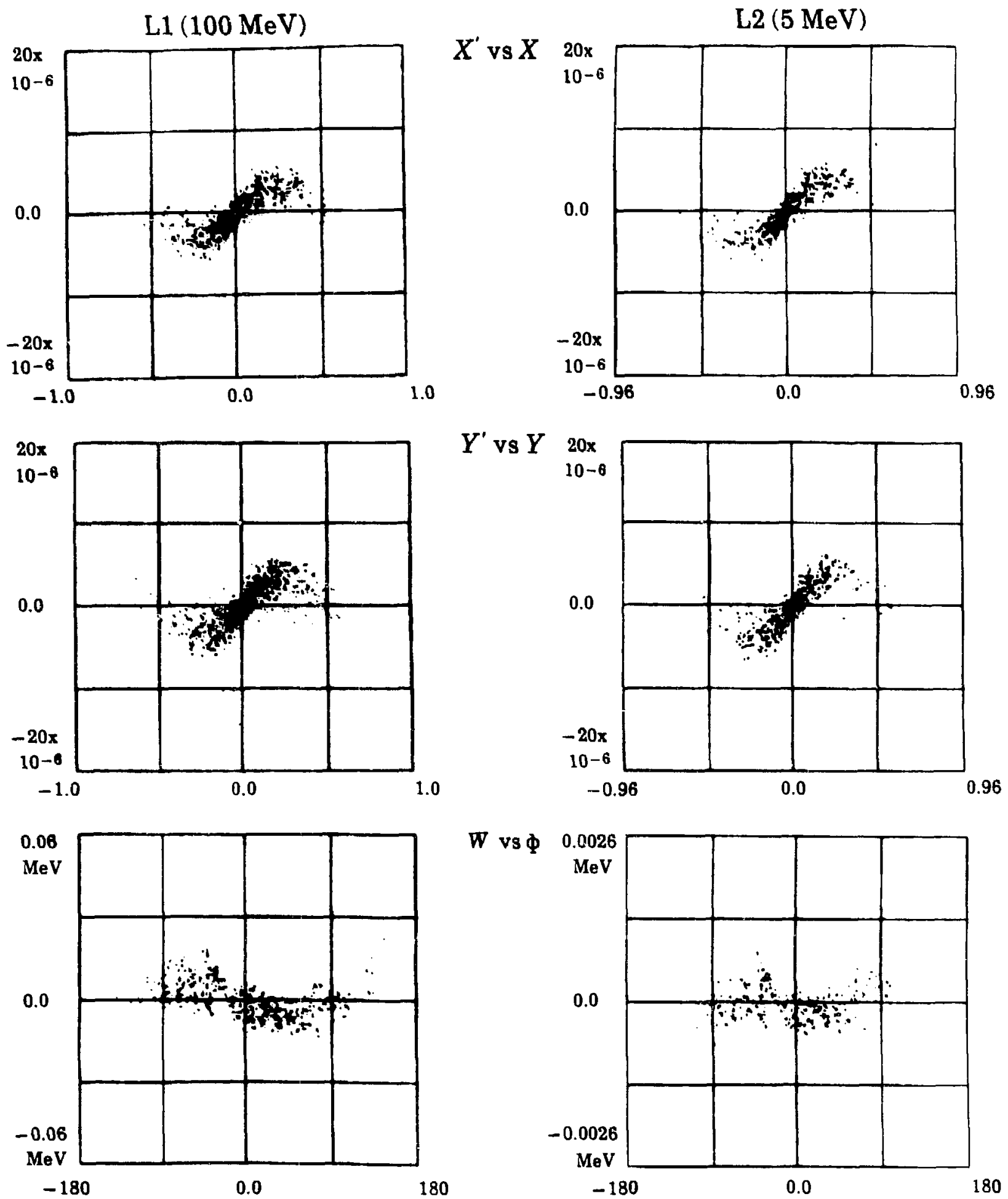

Fig. 1. Scaling law verification example. 
full current beam in transiting the telescope, whereas, there is no emittance growth for a zero current beam. This indicates that space-charge forces are significant in this example.

When using the scaling rules with the various simulation programs, care must be exercised in scaling the various input quantities. For instance, the scaling parameter for a quadrupole characterized by its magnetic field gradient is the ratio of the pole-tip field divided by the aperture radius. Similarly, the scaling of the input beam distribution parameters depends on the parameter definition, which determines the appropriate use of Eqs. (60) through (66).

\section{Conclusion}

The scaling relations derived in this paper show how one beam-transport system can simulate or model the dynamics in another system. The degree of validity of these simulations depends on the degree of validity of the paraxial approximation, how close the simulated beam moment distribution agrees with the actual beam distribution, and how well the individual transport elements are scaled. The EXAMPLE demonstrates one case where the scaling relations perform very well.

\section{ACKNOWLEDGMENTS}

It is a pleasure to thank Walter Lysenko, Barbara Blind and Andrew Jason for their many useful suggestions and comments concerning this work and Robert Glucksterii for his comments pertaining to an early verrion of this work.

\section{REFERENCES}

[1] T. P. Wangler, R. S. Mills, and K. R. Crandall, "Emittance Growth in Intense Beams," Proc. 1987 Particle Accelerator Conf., E. R. Lindstrom and L. S. Taylor, Eds. (IEEE, Washington, DC), Vol. 2, p. 1006.

[2] W. P. Lysenko, "Beam Size and Space Charge," Los Alamos National Laboratory technical memo AT-6:ATN-87-3. 


\section{APPENDIX A}

\section{CODE INPUT}

The purpose of this section is to show how one linear accelerator (linac L1) output optics design can be simulated using PARMILA on a different linac (L2). Both linacs are characterized by their frequencies and output energies. The output beam from linac L1 is characterized by its current, transverse and longitudinal emittances, and Twiss parameters. The beam-transport line elements considered here include magnetic dipoles, quadrupoles, and if bunchers. The scaling of other transport elements follows by analogy. The scaling laws indicate how to transform the beam and transport line from L1 to L2.

The linacs define the time and velocity scales. We obtain the length scaling from Eq. (62):

$$
L_{L 2}=L_{L 1} \frac{v_{L 2} \tau_{L 2}}{v_{L 1} \tau_{L 1}}=L_{L 1} \frac{\beta_{L 2} \lambda_{L 2}}{\beta_{L 1} \lambda_{L 1}}
$$

where $\lambda$ is the linac if free-space wavelength. The scaled current $(I)$, electric pole-tip field $\left(E_{0}\right)$, magnetic pole-tip field ( $B_{0}$, which is also the magnetic dipole field), and the transverse and longitudinal normalized emittance $\left(\epsilon_{n}\right)$ are calculated from Eqs. (63) through (66) and Eq. (67) and are

$$
\begin{aligned}
I_{L 2} & =I_{L 1} \frac{\gamma_{L 2}^{3} \beta_{L 2}^{3}}{\gamma_{L 1}^{3} \beta_{L 1}^{3}} \\
E_{0_{L 2}} & =E_{0_{L 1} 1} \frac{m_{0_{L 2}} L_{L 1} \gamma_{L 2}^{p} \beta_{L 2}^{2}}{m_{0_{L 1}} L_{L 2} \gamma_{L 1}^{p} \beta_{L 1}^{2}}=E_{0_{L 1}} \frac{m_{0_{L 2}} \lambda_{L 1} \gamma_{L 2}^{p} \beta_{L 2}}{m_{0_{L 1}} \lambda_{L 2} \gamma_{L 1}^{p} \beta_{L 1}} \\
B_{0_{L 2}} & =B_{0_{L 1}} \frac{m_{0_{L 2}} L_{L 1} \gamma_{L 2}^{p} \beta_{L 2}}{m_{0_{L 1}} L_{L 2} \gamma_{L 1}^{p} \beta_{L 1}}=B_{0_{L 1}} \frac{m_{0_{L 2}} \lambda_{L 1} \gamma_{L 2}^{p}}{m_{0_{21}} \lambda_{L 2} \gamma_{L 1}^{p}}
\end{aligned}
$$

and

$$
\varepsilon_{\text {norm }_{L 2}}=\varepsilon_{\text {norm }} \frac{L_{L 1} \gamma_{L 2}^{p} \beta_{L 2}}{L_{L 2} \gamma_{L 1}^{p} \beta_{L 1}}=\varepsilon_{\text {norm }_{L 1}} \frac{\lambda_{L 2} \gamma_{L 2}^{p} \beta_{L 2}^{2}}{\lambda_{L 1} \gamma_{L 1}^{p} \beta_{L 1}^{2}}
$$

where the power $p=(1,3)$ for (transverse, longitudinal) fields. PARMILA requires the magnetic field gradient $\left(B_{Q}\right)$ for quadrupoles. Because the quadrupole pole-tip field is the product of the field gradient and bore radius, we have, using Eqs. (67) and (70),

$$
B_{Q_{L 2}}=B_{Q_{L 1}} \frac{m_{0_{L 2}} \lambda_{L 1}^{2} \gamma_{L 2} \beta_{L 1}}{m_{0_{L 1}} \lambda_{L 2}^{2} \gamma_{L 1} \beta_{L 2}}
$$


For bunchers, PARMILA requires the rf potential $(V)$ rather than the electric field. Because $V=E_{0} L$ (the transit time factor is the same in all scaled systems), we have

$$
V_{\text {buncher } L_{2}}=V_{\text {buncher } L_{1}} \frac{m_{0_{L 2}} \gamma_{L 2}^{3} \beta_{L 2}^{2}}{m_{0_{L 1}} \gamma_{L 1}^{3} \beta_{L 1}^{2}}
$$

[A buncher produces a longitudinal field, which is the reason we used $p=3$ in Eq. (73).]

The PARMILA beam input line requires the transverse and longitudinal Twiss parameters ( $\left.\alpha_{\text {Twiss }}, \beta_{\text {Twiss }}\right)$ and the laboratory emittances. PARMILA defines the laboratory emittance $\left(\epsilon_{P}\right)$ as the product of the beam size times the ratio of the momentum spread divided by the nominal momentum. The scaling laws treat the laboratory emittance $\left(\varepsilon_{l a b}\right)$ as the product of the beam size times the ratio of the velocity spread divided by the nominal velocity. For transverse motion, the velocity and momentum ratios are equal. For longitudinal motion, we use Eq. (37). With these considerations, we find that

$$
\epsilon_{P_{L 2}}=\epsilon_{P_{L 1}} \frac{L_{L 2} \gamma_{L 2}^{p-1}}{L_{L 1} \gamma_{L 1}^{p-1}}=\Delta U \frac{\Delta P_{U}}{P_{0}} \quad U=(X, Y, Z)
$$

w'tere the power ' $p$ ' is defined above.

The parameter $\alpha_{T w i s s}$ is dimensionless, therefore,

$$
\alpha_{T w i s s_{L 2}}=\alpha_{T w i s s_{L 1}}
$$

We obtain the $\beta_{\text {Twiss }}$ parameter by observing that the beam size $x$ is related to $\beta_{\text {Twiss }}$ and $\epsilon_{P}$ by

$$
x=\left(\beta_{T w i s s} \epsilon_{P}\right)^{1 / 2} .
$$

Therefore,

$$
\beta_{T w i s s_{L 2}}=\beta_{T w i s s_{L 1}} \frac{L_{L 2} \gamma_{L 1}^{p-1}}{L_{L 1} \gamma_{L 2}^{p-1}} .
$$

The PARMILA output is also scaled in a similar manner. In making Fig. (1), the plut coordinates were scaled. We use Eq. (67) to scale the transverse lengths. The transverse beam divergence and the longitudinal phase spread are the same in all scaled systerris.

PARMILA plots the longitudinal energy spread and we use Eqs. (37) through (39) to obtain

$$
\Delta E_{L 2}=\Delta E_{L 1} \frac{\gamma_{L 2}^{3} \beta_{L 2}^{2}}{\gamma_{L 1}^{3} \beta_{L 1}^{2}}
$$

to scale the energy coordinates. Equations (67) through (78) wern used to scale the PARMILA simulations for the EXAMPLE. 


\section{APPENDIX B}

\section{SOLENOID ScALING}

We show that Eq. (65) is valid for scaling the field of a solenoid magnet. We consider the dynamics of a particle entering a solenoid parallel to the solenoid axis displaced by a distance $r$ from the axis with a velocity $\vec{v}=v_{0} \hat{k}$, where $\hat{k}$ is the unit vector in the axial direction. The solenoid fringe field gives the particle a transverse velocity that couples to the longitudinal magnetic field in the homogenous region of the magnet to produce a spiral motion. We study particle motion in both the fringe field and hornogenous regions of the magnet.

Let $B_{0}$ be the solenoid field strength in the central uniform field region of the magnet; $v_{\perp}$, the transverse particle velocity (obtained in traversing the fringe field); $Z$, the axial distance along the magnet; and $\theta$, the azimuthal position of a particle in the magnet where $\theta=0$ defines the $\mathrm{x}$ axis. We equate the centrifugal and magnetic-field-induced forces in the uniform field region to obtain

$$
v_{\perp}=\frac{e \rho B_{0}}{\gamma m_{0}},
$$

where $\rho$ is the gyroradius. Let $\Delta Z=v_{0} \Delta t, \Delta \theta=v_{\perp} \Delta t / \rho$, and use Eq. (79) to obtain

$$
\Delta \theta=\frac{v_{\perp} \Delta Z}{v_{0} \rho}=\frac{e B_{0} \Delta Z}{\gamma m_{0} v_{0}}
$$

where $\Delta \theta$ is the particles angular displacement in traveling the distance $\Delta Z$ inside the solenoid. The scaling invariant we require, $\Delta \theta / \Delta Z_{n}$, is [see Eq. (14) for the normalization]

$$
\frac{\Delta \theta}{\Delta Z_{n}}=\frac{c B_{0} L}{m_{0} \gamma \beta c}
$$

which is the same as the $B_{0}$ scaling in Eq. (65).

We now consider the particle dynamics in the solenoid fringe field where a particle develops its transverse velocity. Use Maxwell's equation $\nabla \cdot \vec{B}=0$, assume azimuthal symmetry, and expand $B_{r}$ (the radial magnetic field) in a Taylor series keeping only the first-order term $\left[B_{r} \approx r B(Z)\right]$ to obtain

and

$$
\frac{1}{r} \frac{\partial\left(r B_{r}\right)}{\partial r}=-\frac{\partial B_{z}}{\partial Z}
$$

$$
B_{r}=-\frac{r}{2} \frac{\partial B_{z}}{\partial Z}
$$


The equation of motion of a particle through the fringe field assuming the paraxial approximation Eq. (12), making an impulse approximation, and recognizing that $\cos \theta=y / r$ is

$$
\begin{aligned}
\gamma m_{0} \frac{d v_{x}}{d t} & =\gamma m_{0} v_{\mathrm{c}} \frac{d v_{x}}{d Z} \\
& =-e v_{0} B_{r} \sin \theta \\
& =-e v_{0} B_{r} Y / r \\
& =\frac{e v_{0} Y}{2} \frac{\partial B_{z}}{\partial Z}
\end{aligned}
$$

from which we obtain

$$
\begin{aligned}
\Delta v_{x} & =\int d v_{x}=\int_{0}^{B_{0}} \frac{e Y}{2 \gamma m_{0}} d B_{z} \\
& =\frac{e Y B_{0}}{2 \gamma m_{0}}
\end{aligned}
$$

We calculate

$$
\Delta X^{\prime}=\frac{v_{x}}{v_{z}}=\frac{e Y_{n}}{2 c} \frac{B_{0} L}{m_{0} \gamma \beta} .
$$

Because $\Delta X^{\prime}$ and $Y_{n}$ are the same in all scaled systems, Eq. (85) gives the same scaling as Eq. (65). 